\title{
Resposta do arroz irrigado à adubação verde e química no Estado de Tocantins
}

\author{
Nand K. Fageria ${ }^{1}$ \& Alberto B. dos Santos ${ }^{1}$
}

\begin{abstract}
RESUMO
O arroz irrigado é uma cultura significativa para o País, do ponto de vista socioeconômico, em especial nas várzeas tropicais do Estado do Tocantins. Muitas vezes, devido ao uso de adubação inadequada, sua produtividade é limitada. Conduziram-se dois experimentos de campo, na Fazenda Xavante, município de Dueré, Estado do Tocantins, durante dois anos consecutivos, para se avaliar a resposta do arroz irrigado à adubação verde e química, cultivado em um Inceptissolo, cujos tratamentos consistiram de oito combinações de adubação: Adubo verde $+\mathrm{N}_{0} \mathrm{P}_{0} \mathrm{~K}_{0}$; Adubo verde $+\mathrm{N}_{100} \mathrm{P}_{120} \mathrm{~K}_{100} ;$ Adubo verde $+\mathrm{N}_{50} \mathrm{P}_{60} \mathrm{~K}_{50}$; Adubo verde $+\mathrm{N}_{75} \mathrm{P}_{90} \mathrm{~K}_{75}$; Adubo verde $+\mathrm{N}_{45} \mathrm{P}_{120} \mathrm{~K}_{100}$ (aplicação de $\mathrm{N}$, $\mathrm{P}$ e K no plantio); Adubo verde $+\mathrm{N}_{45} \mathrm{P}_{120} \mathrm{~K}_{100}$ (aplicação de P e K no plantio e N em cobertura); Adubo verde + $\mathrm{N}_{100} \mathrm{P}_{120} \mathrm{~K}_{100}+4 \mathrm{Mg}$ ha-1 calcário; e Sem adubo verde $+\mathrm{N}_{100} \mathrm{P}_{120} \mathrm{~K}_{100}$. Obteve-se a produtividade máxima (kg ha-1) de grãos com o tratamento adubo verde $+\mathrm{N}_{45} \mathrm{P}_{120} \mathrm{~K}_{100}$ (aplicação de $\mathrm{P}$ e $\mathrm{K}$ no plantio e $\mathrm{N}$ em cobertura). Entre os componentes da produtividade, o comprimento da panícula foi influenciado positiva e significativamente, pelos tratamentos de adubação. Com a prática da adubação verde, a quantidade de fertilizante nitrogenado a ser empregada pode ser reduzida e, em conseqüência, diminuir também o custo de produção do arroz irrigado.
\end{abstract}

Palavras-chave: solo de várzea, Oryza sativa, disponibilidade de nutrientes, produtividade de grãos

\section{Response of irrigated rice to green manure and chemical fertilization in the State of Tocantins}

\begin{abstract}
Lowland rice is an important crop in Brazil due to its socio-economic value. Often due to inadequate fertilization, production is limited. Two field experiments were conducted at Xavante Farm, Municipality of Dueré, State of Tocantins, Brazil, during two consecutive years to determine the response of irrigated rice to green manure and to chemical fertilization grown in an Inceptisol. The treatments consisted of green manure $+\mathrm{N}_{0} \mathrm{P}_{0} \mathrm{~K}_{0}$; green manure $+\mathrm{N}_{100} \mathrm{P}_{120} \mathrm{~K}_{100}$; green manure $+\mathrm{N}_{50} \mathrm{P}_{60} \mathrm{~K}_{50}$; green manure $+\mathrm{N}_{75} \mathrm{P}_{90} \mathrm{~K}_{75}$; green manure $+\mathrm{N}_{45} \mathrm{P}_{120} \mathrm{~K}_{100}$ (application of $\mathrm{N}$, $\mathrm{P}$, and $\mathrm{K}$ at sowing); green manure $+\mathrm{N}_{45} \mathrm{P}_{120} \mathrm{~K}_{100}$ (application of $\mathrm{P}$ and $\mathrm{K}$ at sowing and $\mathrm{N}$ topdressing); green manure $+\mathrm{N}_{100} \mathrm{P}_{120} \mathrm{~K}_{100}$ $+4 \mathrm{Mg}$ lime ha- ${ }^{-1}$; and without green manure $+\mathrm{N}_{100} \mathrm{P}_{120} \mathrm{~K}_{100}$. Maximum grain yield was obtained with the application of green manure $+\mathrm{N}_{45} \mathrm{P}_{120} \mathrm{~K}_{100}$ (application of $\mathrm{P}$ and $\mathrm{K}$ at sowing and $\mathrm{N}$ topdressing). Among yield components, panicle length was significantly and positively influenced by fertilization. These results show that green manure can reduce chemical fertilization, and hence cost of production.
\end{abstract}

Key words: lowland soil, Oryza sativa, nutrient uptake, grain yield 


\section{INTRODUÇÃO}

Dentre as culturas anuais, o arroz é a mais consumida no mundo, devido à dieta básica de mais de $50 \%$ da população mundial. A maior área e consumo de arroz estão no continente asiático, sendo a China e a Índia seus maiores produtores e consumidores. A importância da cultura do arroz para o Brasil aumenta, juntamente com o feijão, na medida em que cresce o contingente populacional interno; aqui, ele é cultivado em dois ecossistemas denominados várzeas e terras altas, sob diversos sistemas de plantio. O ecossistema de arroz irrigado contribui com aproximadamente 68\% da produção e $41 \%$ da área plantada no País.

No processo de modernização e racionalização da agricultura brasileira, o uso de adubação adequada constitui fator imprescindível para o aumento da produtividade (Raij, 1991). O custo crescente dos insumos agrícolas exige cada vez mais a adoção de métodos e técnicas de cultivo próprios para a produção das culturas anuais, como o arroz, enquanto o uso racional da adubação não aumenta apenas a produtividade mas, diminui o custo de produção e os riscos de poluição ambiental.

A adubação verde é a prática de se incorporar, ao solo, o tecido vegetal não decomposto, visando manter ou aumentar a fertilidade do solo. A importância da adubação verde no aumento da produtividade das culturas subseqüentes já é reconhecida pelos agricultores, desde $500 \mathrm{aC}$. Os agricultores do sul e sudeste da Ásia vêm praticando a adubação verde em arroz há longo tempo porém sua ênfase decresceu com a introdução dos sistemas agrícolas intensivos e a disponibilidade de fertilizantes químicos (Singh et al., 1991). Os movimentos atuais para o uso reduzido de insumos e o aumento do uso dos sistemas de produção biológicos, têm renovado o interesse no uso de adubação verde com leguminosas, como fonte de N (Fageria et al., 1999); neste sentido, a avaliação do efeito da adubação verde e da adubação química é fundamental para o manejo da fertilidade do solo na produção de culturas anuais, como o arroz irrigado (Fage- ria, 2000). O conhecimento do efeito da adubação verde junto com adubação química é de grande relevância, considerando-se a necessidade de racionalização do uso de fertilizantes e de aumentar a eficiência nutricional da cultura de arroz irrigado. Devido as informações sobre este assunto serem escassas, conduziram-se experimentos de campo com o objetivo de se avaliar os efeitos da adubação verde em cojunto com o adubo químico sobre a produtividade de arroz irrigado, em solo de várzea.

\section{MATERIAL E MÉTODOS}

Dois experimentos de campo foram conduzidos na Fazenda Xavante, município de Dueré, Estado do Tocantins, durante dois anos consecutivos. O solo das áreas experimentais é classificado como Glei pouco húmico de várzea (Inceptissolo). Os resultados das análises química e granulométrica das amostras dos solos das áreas experimentais são apresentadas na Tabela 1.

Os experimentos foram designados como 1 e 2. Os P, K, $\mathrm{Zn}, \mathrm{Cu}, \mathrm{Fe}$ e Mn e extraídos pelo extrator Mehlich 1 (0,05 $\mathrm{M} \mathrm{HCl}+0,0125 \mathrm{M} \mathrm{H}_{2} \mathrm{SO}_{4}$ ) e os $\mathrm{O}$, Ca, $\mathrm{Mg}$ e o $\mathrm{Al}$ foram com solução de $\mathrm{KCl} 1 \mathrm{M}$. O P foi determinado por colorímetro, o K por fotômetro de chama, os $\mathrm{Cu}$, Zn, Fe e Mn por absorção atômica, o Ca e Mg por titulação com EDTA e o Al por titulação com $\mathrm{NaOH}$. Determinou-se a matéria orgânica através do método de Walkley-Black. A metodologia de análise está descrita no Manual de Métodos de Análise de Solo, da Embrapa (1997).

Os tratamentos consistiram de oito combinações de adubação: 1) Adubo verde $+\mathrm{N}_{0} \mathrm{P}_{0} \mathrm{~K}_{0}$; 2) Adubo verde $+\mathrm{N}_{100} \mathrm{P}_{120} \mathrm{~K}_{100}$; 3) Adubo verde $+\mathrm{N}_{50} \mathrm{P}_{60} \mathrm{~K}_{50}$; 4) Adubo verde $+\mathrm{N}_{75} \mathrm{P}_{90} \mathrm{~K}_{75}$; 5) Adubo verde $+\mathrm{N}_{45} \mathrm{P}_{120} \mathrm{~K}_{100}$ (aplicação de $\mathrm{N}$, P e K no plantio); 6) Adubo verde $+\mathrm{N}_{45} \mathrm{P}_{120} \mathrm{~K}_{100}$ (aplicação de $\mathrm{P}$ e $\mathrm{K}$ no plantio e $\mathrm{N}$ em cobertura); 7) Adubo verde $+\mathrm{N}_{100} \mathrm{P}_{120} \mathrm{~K}_{100}+$ $4 \mathrm{Mg} \mathrm{ha}^{-1}$ calcário e 8) Sem adubo verde $+\mathrm{N}_{100} \mathrm{P}_{120} \mathrm{~K}_{100}$. No segundo ano, o arroz foi plantado nas mesmas parcelas sem

Tabela 1. Propriedades químicas e granulometria das amostras de solo das áreas experimentais antes da aplicação dos tratamentos de adubação, produção de matéria seca da parte aérea de mucuna e acumulação de nutrientes

\begin{tabular}{|c|c|c|c|}
\hline $\begin{array}{l}\text { Propriedades do solo } \\
\text { (Experimento 1) }\end{array}$ & $\begin{array}{l}\text { Propriedades do solo } \\
\text { (Experimento 2) }\end{array}$ & $\begin{array}{l}\text { Matéria seca/nutriente } \\
\text { (Experimento } 1)^{1}\end{array}$ & $\begin{array}{l}\text { Matéria seca/nutriente } \\
\text { (Experimento 2) }\end{array}$ \\
\hline $\mathrm{pH}$, em $\mathrm{H}_{2} \mathrm{O}(5,6)$ & $\mathrm{pH}$ em $\mathrm{H}_{2} \mathrm{O}(5,7)$ & MS, kg ha-1 (6930) & MS, kg ha-1 (7102) \\
\hline $\mathrm{P}, \mathrm{mg} \mathrm{dm} \mathrm{dm}^{-3}(9,7)$ & $\mathrm{P}, \mathrm{mg} \mathrm{dm}^{-3}(12,0)$ & $\mathrm{N}, \mathrm{kg} \mathrm{ha}^{-1}(136,3)$ & $\mathrm{N}, \mathrm{kg} \mathrm{ha}^{-1}(234,1)$ \\
\hline $\mathrm{K}, \mathrm{mg} \mathrm{dm}^{-3}(97)$ & $\mathrm{K}, \mathrm{mg} \mathrm{dm}^{-3}(120)$ & P, kg ha-1 $(9,2)$ & P, kg ha ${ }^{-1}(14,1)$ \\
\hline $\mathrm{Ca}, \mathrm{cmol}_{\mathrm{c}} \mathrm{dm}^{-3}(1,6)$ & $\mathrm{Ca}, \mathrm{cmol}_{\mathrm{c}} \mathrm{dm}^{-3}(1,7)$ & $\mathrm{K}, \mathrm{kg} \mathrm{ha}^{-1}(122,4)$ & K, kg ha-1 $(199,8)$ \\
\hline $\mathrm{Mg}, \mathrm{cmol}_{\mathrm{c}} \mathrm{dm}^{-3}(0,9)$ & $\mathrm{Mg}, \mathrm{cmol}_{\mathrm{c}} \mathrm{dm}^{-3}(0,9)$ & $\mathrm{Ca}, \mathrm{kg} \mathrm{ha}^{-1}(57,3)$ & $\mathrm{Ca}, \mathrm{kg} \mathrm{ha}^{-1}(88,66)$ \\
\hline $\mathrm{Al}, \mathrm{cmol}_{\mathrm{c}} \mathrm{dm}^{-3}(0,3)$ & $\mathrm{Al}, \mathrm{cmol}_{\mathrm{c}} \mathrm{dm}^{-3}(0,2)$ & $\mathrm{Mg}, \mathrm{kg} \mathrm{ha}^{-1}(13,1)$ & $\mathrm{Mg}, \mathrm{kg} \mathrm{ha}^{-1}(24,2)$ \\
\hline $\mathrm{Cu}, \mathrm{mg} \mathrm{dm}^{-1}(2,3)$ & $\mathrm{Cu}, \mathrm{mg} \mathrm{dm}^{-1}(2,0)$ & Zn, g ha-1 $(174,3)$ & $\mathrm{Zn}, \mathrm{g} \mathrm{ha}^{-1}(284,4)$ \\
\hline $\mathrm{Zn}, \mathrm{mg} \mathrm{dm}^{-1}(4,2)$ & $\mathrm{Zn}, \mathrm{mg} \mathrm{dm}^{-1}(3,5)$ & $\mathrm{Cu}, \mathrm{g} \mathrm{ha}^{-1}(127,0)$ & $\mathrm{Cu}, \mathrm{g}$ ha $^{-1}(213,0)$ \\
\hline $\mathrm{Fe}, \mathrm{mg} \mathrm{dm}^{-1}(319)$ & $\mathrm{Fe}, \mathrm{mg} \mathrm{dm}^{-1}(341)$ & Mn, g ha-1 $(1280,9)$ & Mn, g ha-1 $(2054,3)$ \\
\hline $\mathrm{Mn}, \mathrm{mg} \mathrm{kg}^{-1}(46)$ & $\mathrm{Mn}, \mathrm{mg} \mathrm{kg}^{-1}(45)$ & $\mathrm{Fe}, \mathrm{g} \mathrm{ha}^{-1}(1806,4)$ & $\mathrm{Fe}, \mathrm{g} \mathrm{ha}^{-1}(3249,1)$ \\
\hline M.0., g dm${ }^{-3}(12,0)$ & M. 0., g dm${ }^{-3}(11,0)$ & & \\
\hline Argila, $\mathrm{g} \mathrm{dm}^{-3}(350)$ & Argila, $\mathrm{g} \mathrm{dm}^{-3}$ (305) & & \\
\hline Silte, $\mathrm{g} \mathrm{dm}^{-3}(90)$ & Silte, $\mathrm{g} \mathrm{dm}^{-3}$ (125) & & \\
\hline Areia, $\mathrm{g} \mathrm{dm}^{-3}(560)$ & Areia, $\mathrm{g} \mathrm{dm}^{-3}(570)$ & & \\
\hline
\end{tabular}

${ }^{1} 0$ s valores são médias de sete parcelas e três repetições 
adubo verde mas com as mesmas doses de adubo químico do primeiro cultivo, com exceção de calcário, a adubação no plantio foi aplicada manualmente e misturada a sachos no sulco; já o nitrogênio, o fósforo e o potássio foram aplicados na forma de uréia, superfosfato triplo e cloreto de potássio, respectivamente. Todas as adubações foram aplicadas no sulco de plantio, por ocasião da semeadura, nos dois anos de cultivo, com exceção do $\mathrm{N}$, que foi parcelado, em cujos tratamentos 2, 3, 4, 7 e 8, metade do $\mathrm{N}$ foi aplicado no plantio e o restante no estádio de perfilhamento ativo.

O calcário utilizado continha $\mathrm{CaO}$ 27,4\%, MgO 17,2\% e PRNT 72,3\%; sua dose foi calculada com base no teor de Al e $\mathrm{Ca}+\mathrm{Mg}$ trocáveis, utilizados na seguinte fórmula:

Dose de calcário $\left(\mathrm{Mg} \mathrm{ha}^{-1}\right)=\left(2 \mathrm{X} \mathrm{Al}^{3+}\right)+\left\{2-\left(\mathrm{Ca}^{2+}+\mathrm{Mg}^{2+}\right)\right\}$

O adubo verde foi a Mucuna cinza (Mucuna cinereum) com $60 \mathrm{~kg}$ semente ha-1, no espaçamento de $50 \mathrm{~cm}$ entre fileiras e incorporado ao solo 91 dias após a semeadura; a primeira cultura de arroz foi plantada 56 dias após a sua incorporação. Na época da incorporação do adubo verde, retirou-se uma amostra de plantas desse adubo em $1 \mathrm{~m}$ da fileira de plantio, em cada parcela, para determinação da matéria seca e análise química das plantas (Tabela 1).

O delineamento experimental usado foi de blocos casualizados, com três repetições; o tamanho de cada parcela foi de $3 \times 5 \mathrm{~m}$. A cultivar de arroz plantada foi a Epagri 109, com 100 sementes por metro, no espaçamento de $0,20 \mathrm{~m}$ entre fileiras. Colheram-se as fileiras centrais de cada parcela deixando-se $0,5 \mathrm{~m}$ nas extremidades e duas fileiras laterais como bordadura, coletando-se 11 fileiras centrais. Na época da colheita colheram-se plantas em $1 \mathrm{~m}$ de extensão na fileira em cada parcela (somente no ensaio 1) para determinar a produção de matéria seca da parte aérea e grãos e realizar a análise química. Para determinação dos teores de nutrientes as plantas foram secadas em estufa a $70{ }^{\circ} \mathrm{C}$, moídas e digeridas com mistura de ácidos nítrico e perclórico 2:1. Para análise do N, o material foi digerido com ácido sulfúrico. A metodologia utilizada para as análises químicas das plantas foi descrita por Moraes \& Rabelo (1986). Após a colheita efetuou-se amostragem do solo a $0-20 \mathrm{~cm}$ de profundidade (somente no experimento 1 ), coletando-se 40 subamostras ao acaso, em cada parcela.

Os dados foram submetidos a análise de variância e as médias dos tratamentos comparadas entre si pelo teste de Tukey, a 5\% de probabilidade usou-se também, regressão polinomial na análise além de interpretação de alguns resultados.

\section{RESULTADOS E DISCUSSÃO}

A produtividade de grãos sob os diferentes tratamentos de adubação, são apresentadas na Tabela 2. Não houve interação significativa entre ano e adubação; portanto, os dados médios dos dois cultivos se acham nos dois experimentos. A produtividade de grãos foi significativamente influenciada pelos tratamentos de adubação, nos dois experimentos. Na análise conjunta dos dois experimentos constataram-se diferenças significativas para os tratamentos de adubação e ensaios.
Tabela 2. Produtividade de grãos $\left(\mathrm{kg} \mathrm{ha}^{-1}\right)$ do arroz sob diferentes tratamentos de adubação

\begin{tabular}{lccl}
\hline Tratamentos & Experimento 1 Experimento 2 & Média \\
Ad.V. $+\mathrm{N}_{0} \mathrm{P}_{0} \mathrm{~K}_{0}$ & $4778 \mathrm{c}$ & $3982 \mathrm{e}$ & $4380 \mathrm{c}$ \\
Ad.V. $+\mathrm{N}_{100} \mathrm{P}_{120} \mathrm{~K}_{100}$ & $5070 \mathrm{bc}$ & $5224 \mathrm{a}$ & $5147 \mathrm{abc}$ \\
Ad.V. $+\mathrm{N}_{50} \mathrm{P}_{60} \mathrm{~K}_{50}$ & $5027 \mathrm{bc}$ & $4286 \mathrm{de}$ & $4657 \mathrm{bc}$ \\
Ad.V. $+\mathrm{N}_{75} \mathrm{P}_{90} \mathrm{~K}_{75}$ & $5651 \mathrm{ab}$ & $5090 \mathrm{ab}$ & $5370 \mathrm{ab}$ \\
$\begin{array}{l}\text { Ad.V. }+\mathrm{N}_{45} \mathrm{P}_{120} \mathrm{~K}_{100} \text { (N, P e K no } \\
\text { plantio) }\end{array}$ & $5129 \mathrm{bc}$ & $4910 \mathrm{abc}$ & $5020 \mathrm{bc}$ \\
$\begin{array}{l}\text { Ad. V. }+\mathrm{N}_{45} \mathrm{P}_{120} \mathrm{~K}_{100} \text { (P e K no } \\
\text { plantio e N em cobertura) }\end{array}$ & $5904 \mathrm{a}$ & $5179 \mathrm{ab}$ & $5542 \mathrm{a}$ \\
Ad.V. $+\mathrm{N}_{100} \mathrm{P}_{120} \mathrm{~K}_{100}+4 \mathrm{Mg}$ & $4847 \mathrm{c}$ & $4659 \mathrm{bcd}$ & $4753 \mathrm{bc}$ \\
calcario ha ${ }^{-1}$ & $4870 \mathrm{c}$ & $4527 \mathrm{~cd}$ & $4699 \mathrm{bc}$ \\
Sem Ad. V. $+\mathrm{N}_{100} \mathrm{P}_{120} \mathrm{~K}_{100}$ & 5160 & 4732 & 4946 \\
Média & $* *$ & $\mathrm{~ns}$ & $* *$ \\
Teste F (Ano) & $*$ & $\star *$ & $* *$ \\
Teste F (Adubação) & $\mathrm{ns}$ & $\mathrm{ns}$ & $\mathrm{ns}$ \\
Teste F (Ano X Adubação) & 11,6 & 9,0 & 7,9 \\
C.V. (\%) &
\end{tabular}

*, ${ }^{* *}$, ns Significativo a $5 \%$ e $1 \%$ de probabilidade e não significativo, respectivamente. Médias seguidas da mesma letra, na mesma coluna, não diferem significativamente pelo teste de Tukey, a $5 \%$ de probabilidade

Considerando-se a média dos dois experimentos, a produtividade máxima $\left(\mathrm{kg} \mathrm{ha}^{-1}\right)$ foi obtida no tratamento adubação verde $+\mathrm{N}_{45} \mathrm{P}_{120} \mathrm{~K}_{100}$ (P e K no plantio e $\mathrm{N}$ em cobertura). $\mathrm{O}$ aumento na produtividade sob este tratamento foi de $26 \%$ em comparação com o tratamento adubação verde $+\mathrm{N}_{0} \mathrm{P}_{0} \mathrm{~K}_{0}$. Em solos de várzea intensivamente cultivados, a importância da adubação para a cultura de arroz irrigado tem sido relatada por vários autores (Santos et al., 2002; Mariot et al., 2003; Fageria et al., 2003). Verifica-se que a média dos dois experimentos do tratamento adubação verde $+\mathrm{N}_{45} \mathrm{P}_{120} \mathrm{~K}_{100}$ (P e K no plantio e $\mathrm{N}$ em cobertura) produziu $8 \%$ mais grãos que o tratamento adubação verde + $\mathrm{N}_{100} \mathrm{P}_{120} \mathrm{~K}_{100}$, isto é, uma economia de $55 \mathrm{~kg} \mathrm{~N} \mathrm{ha}^{-1}$ na produção de arroz irrigado. Kolar et al. (1993) relataram que a adubação verde pode substituir $60 \mathrm{~kg} \mathrm{~N} \mathrm{ha}^{-1}$ na cultura de arroz irrigado.

A comparação da produtividade de grãos entre os tratamentos com adubação verde $+\mathrm{N}_{100} \mathrm{P}_{120} \mathrm{~K}_{100}$, adubação química recomendada pela pesquisa para a cultura do arroz irrigado (Fageria et al., 2003), e sem adubação verde $+\mathrm{N}_{100} \mathrm{P}_{120} \mathrm{~K}_{100}$ indicou aumento de $10 \%$ na produtividade, ressaltando a importância de adubação verde na produção de arroz irrigado. Balasubramaniyan \& Palaniappan (1992) determinaram os efeitos do adubo verde sobre a produtividade de arroz irrigado em um solo franco-argiloso, com aplicação de 12,5 $\mathrm{t} \mathrm{ha}^{-1}$ de Sesbania aculeata; o adubo verde aumentou significativamente a produtividade de grãos de arroz. De acordo com Fageria et al. (1999), a adubação verde, além da fixação de N, melhora as propriedades físicas do solo e fornece nutrientes para as plantas; além disso, reduz a lixiviação de N, controla as plantas daninhas e minimiza a erosão do solo e, conseqüentemente, aumenta a produtividade das culturas (Singh et al., 1991; George et al., 1994).

Avaliaram-se os efeitos da adubação química e adubação verde sobre o número de panículas, comprimento da panícula, massa de 1000 grãos e esterilidade de espiguetas (Tabela 3). 
Tabela 3. Influência dos tratamentos no número de panículas, comprimento da panícula, massa de 1000 grãos e esterilidade de espiguetas de arroz irrigado. Experimento 1

\begin{tabular}{|c|c|c|c|c|c|}
\hline \multirow[t]{2}{*}{ Tratamentos } & \multirow{2}{*}{$\begin{array}{l}\text { Panículas } \\
\left(\mathrm{n}^{0} \mathrm{~m}^{-2}\right)\end{array}$} & \multicolumn{2}{|c|}{$\begin{array}{l}\text { Comprimento da } \\
\text { panícula (cm) }\end{array}$} & \multirow{2}{*}{$\begin{array}{l}\text { Massa de } \\
1000 \\
\text { grãos (g) }\end{array}$} & \multirow{2}{*}{$\begin{array}{l}\text { Ester. de } \\
\text { espiguetas } \\
(\%)\end{array}$} \\
\hline & & $1^{0}$ cultivo & $2^{0}$ cultivo & & \\
\hline Ad.V. $+\mathrm{N}_{0} \mathrm{P}_{0} \mathrm{~K}_{0}$ & 322,5 & $20,1 \mathrm{c}$ & 19,8 b & 26,6 & 13,0 \\
\hline Ad.V. $+\mathrm{N}_{100} \mathrm{P}_{120} \mathrm{~K}_{100}$ & 371,3 & $22,3 a b$ & $23,1 \mathrm{a}$ & 26,7 & 15,6 \\
\hline Ad.V. $+\mathrm{N}_{50} \mathrm{P}_{60} \mathrm{~K}_{50}$ & 364,7 & $22,0 \mathrm{ab}$ & $22,1 \mathrm{a}$ & 26,5 & 13,3 \\
\hline Ad.V. $+\mathrm{N}_{75} \mathrm{P}_{90} \mathrm{~K}_{75}$ & 368,5 & $23,1 \mathrm{a}$ & $21,9 a b$ & 26,7 & 15,1 \\
\hline $\begin{array}{l}\text { Ad.V. }+\mathrm{N}_{45} \mathrm{P}_{120} \mathrm{~K}_{100} \\
\text { (N, P e K no plantio) }\end{array}$ & 378,5 & $23,0 \mathrm{a}$ & $21,8 \mathrm{ab}$ & 26,6 & 12,9 \\
\hline $\begin{array}{l}\text { Ad. V. }+\mathrm{N}_{45} \mathrm{P}_{120} \mathrm{~K}_{100} \\
\text { (P e K no plantio e N em } \\
\text { cobertura) }\end{array}$ & 379,5 & $21,5 \mathrm{bc}$ & $22,8 \mathrm{a}$ & 27,1 & 13,1 \\
\hline $\begin{array}{l}\text { Ad.V. }+\mathrm{N}_{100} \mathrm{P}_{120} \mathrm{~K}_{100}+ \\
4 \text { t calcário ha }\end{array}$ & 365,5 & $22,8 \mathrm{ab}$ & $22,0 \mathrm{ab}$ & 26,7 & 13,7 \\
\hline $\begin{array}{l}\text { Sem Ad. V. + } \\
N_{100} P_{120} K_{100}\end{array}$ & 346,0 & $22,7 \mathrm{ab}$ & $21,9 \mathrm{ab}$ & 25,9 & 13,7 \\
\hline Média & 362,1 & 22,2 & 21,9 & 26,6 & 13,8 \\
\hline Teste F Ano & $\star \star$ & $\star *$ & & ns & * \\
\hline Teste F Adubação & ns & ** & & ns & ns \\
\hline $\begin{array}{l}\text { Teste F } \\
\text { (Ano X Adubação) }\end{array}$ & ns & * & & ns & ns \\
\hline C. V. (\%) & 10,5 & 3,0 & & 2,2 & 17,5 \\
\hline
\end{tabular}

* ** ns Significativo a $5 \%$ e $1 \%$ de probabilidade e não significativo, respectivamente Médias seguidas da mesma letra na mesma coluna não diferem significativamente pelo teste de Tukey, a $5 \%$ de probabilidade

Entre os componentes da produtividade, o comprimento da panícula foi influenciado significativamente pelos tratamentos e, da mesma forma, pelo ano de cultivo, tratamentos de adubação e interação entre ano e adubação, mas no tratamento que não recebeu adubação química ele foi reduzido de forma significativa, em comparação com os demais tratamentos. O número de panículas não foi influenciado significativamente pelos tratamentos de adubação porém o número máximo de panículas foi obtido com o tratamento de adubação verde $+\mathrm{N}_{45} \mathrm{P}_{120} \mathrm{~K}_{100}$ ( $\mathrm{P}$ e $\mathrm{K}$ no plantio e $\mathrm{N}$ em cobertura) e o mínimo com adubação verde $+\mathrm{N}_{0} \mathrm{P}_{0} \mathrm{~K}_{0}$. A maior produtividade de grãos também foi obtida naquele tratamento. O aumento no número de panículas com este tratamento foi de $18 \%$ em comparação com o tratamento de adubação verde $+\mathrm{N}_{0} \mathrm{P}_{0} \mathrm{~K}_{0}$, significando que a adubação quí- mica é significativa no aumento do número de panícula, e em conseqüência na produtividade do arroz irrigado (Fageria \& Baligar, 1996; Fageria et al., 2003). A adubação verde também aumentou a massa de 1000 grãos porém não ocorreu aumento significativo; tais resultados indicam a possibilidade de se incrementar o número de panículas por unidade de área e o comprimento da panícula com adubação orgânica e química e, sem dúvida de aumentar a produtividade do arroz irrigado em solo de várzea. Vários trabalhos mostram que o número de panículas e o comprimento da panícula se correlacionam positivamente com a produtividade de grãos, na cultura do arroz (Gravois \& Helms, 1992; Hasegawa et al., 1994; Fageria et al., 2003).

Apresentam-se, nas Tabelas 4 e 5, os valores médios da acumulação de nutrientes na parte aérea e nos grãos. A acumulação de $\mathrm{N}$, K e Mg na parte aérea e de $\mathrm{N}$ nos grãos, foi influenciada significativamente pelos tratamento de adubação. A acumulação de nutrientes na parte aérea obedeceu a seguinte ordem: $\mathrm{K}>\mathrm{N}>\mathrm{Ca}>\mathrm{Mg}>\mathrm{P}>\mathrm{Mn}>\mathrm{Fe}>\mathrm{Zn}>$ $\mathrm{Cu}$ e, nos grãos, $\mathrm{N}>\mathrm{P}>\mathrm{K}>\mathrm{Mg}>\mathrm{Ca}>\mathrm{Mn}>\mathrm{Fe}>\mathrm{Zn}>$ Cu. Fageria \& Baligar (1996) obtiveram a mesma ordem de acumulação de macro e micronutrientes na parte aérea e nos grãos, na cultura de arroz irrigado. Para produzir uma tonelada de grãos, a cultura de arroz irrigado acumula, na parte aérea e nos grãos, em média $19 \mathrm{~kg}$ de N, $4 \mathrm{~kg}$ de P, $25 \mathrm{~kg}$ de $\mathrm{K}, 5 \mathrm{~kg}$ de Ca, $3 \mathrm{~kg}$ de Mg, 73 g de Zn, $27 \mathrm{~g}$ de $\mathrm{Cu}, 1285 \mathrm{~g}$ de Fe e $442 \mathrm{~g}$ de $\mathrm{Mn}$.

Em relação à distribuição de nutrientes na parte aérea e nos grãos de arroz, em média 52\% do N, 60\% do P, $8 \%$ do K, $16 \%$ do Ca, 33\% do Mg, $45 \%$ do Zn, $84 \%$ do $\mathrm{Cu}, 26 \%$ do $\mathrm{Mn}$ e $33 \%$ do Fe foram acumulados nos grãos e o restante ficou na parte aérea. Fageria (2000) relata a distribuição de macro e micronutrientes nas mesmas proporções, na parte aérea e nos grãos na cultura do arroz irrigado cultivado em solo de várzea.

Determinou-se a relação entre acumulação de nutrientes nos grãos e produtividade de grãos (Tabela 6). Não ocorreu relação significativa entre produtividade de grãos e acumulação de nutrientes na parte aérea; portanto, os dados não foram apresentados. A produtividade de grãos foi influenciada

Tabela 4. Acumulação de nutrientes na parte aérea do arroz irrigado em diferentes tratamentos de adubação. Experimento 1

\begin{tabular}{|c|c|c|c|c|c|c|c|c|c|}
\hline \multirow[t]{2}{*}{ Tratamentos } & $\mathrm{N}$ & $\mathbf{P}$ & K & $\mathrm{Ca}$ & $\mathrm{Mg}$ & $\mathrm{Zn}$ & $\mathrm{Cu}$ & Mn & $\mathrm{Fe}$ \\
\hline & \multicolumn{5}{|c|}{$\mathrm{kg} \mathrm{ha}^{-1}$} & \multicolumn{4}{|c|}{ g ha-1 } \\
\hline Ad.V. $+\mathrm{N}_{0} \mathrm{P}_{0} \mathrm{~K}_{0}$ & $35,6 \mathrm{~b}$ & 6,4 & $91,5 \mathrm{a}$ & 17,7 & $7,6 \mathrm{~d}$ & 156,5 & 16,5 & 4580,2 & 1064,7 \\
\hline Ad.V. $+\mathrm{N}_{100} \mathrm{P}_{120} \mathrm{~K}_{100}$ & $51,2 \mathrm{a}$ & 8,3 & $113,0 a b c$ & 24,3 & $14,1 \mathrm{ab}$ & 225,0 & 20,5 & 5724,0 & 1456,3 \\
\hline Ad.V. $+\mathrm{N}_{50} \mathrm{P}_{60} \mathrm{~K}_{50}$ & $39,2 \mathrm{ab}$ & 7,0 & 95,8 a & 18,0 & $9,5 \mathrm{~cd}$ & 176,5 & 19,7 & 3895,8 & 1431,7 \\
\hline Ad.V. $+\mathrm{N}_{75} \mathrm{P}_{90} \mathrm{~K}_{75}$ & $51,0 \mathrm{a}$ & 7,7 & $121,2 a b c$ & 22,9 & $11,9 a b c$ & 212,0 & 28,5 & 5039,8 & 1383,8 \\
\hline Ad.V. $+\mathrm{N}_{45} \mathrm{P}_{120} \mathrm{~K}_{100}$ (N, P e K no plantio) & $52,0 \mathrm{a}$ & 7,7 & $120,5 a b c$ & 20,9 & 10,3 bcd & 219,7 & 25,7 & 4965,7 & 1686,2 \\
\hline Ad. $V .+N_{45} P_{120} K_{100}$ (P e K no plantio e $N$ em cobertura) & 52,8 a & 9,8 & $141,5 \mathrm{~b}$ & 24,8 & $13,7 \mathrm{ab}$ & 255,5 & 26,3 & 6231,3 & 1877,7 \\
\hline Ad.V. $+\mathrm{N}_{100} \mathrm{P}_{120} \mathrm{~K}_{100}+4 \mathrm{t}$ calcário ha ${ }^{-1}$ & $53,8 \mathrm{a}$ & 8,5 & 139,3 bc & 24,6 & $14,4 \mathrm{a}$ & 198,2 & 22,2 & 4132,3 & 1392,3 \\
\hline Sem Ad. V. $+\mathrm{N}_{100} \mathrm{P}_{120} \mathrm{~K}_{100}$ & $50,6 \mathrm{a}$ & 7,2 & $108,3 a b c$ & 21,4 & 11,1 abcd & 224,2 & 20,0 & 4703,3 & 1946,8 \\
\hline Média & 48,3 & 7,8 & 116,4 & 21,8 & 11,6 & 208,5 & 22,4 & 4909,1 & 1529,9 \\
\hline Teste F Ano & ns & * & ns & $\mathrm{ns}$ & ns & ns & ns & * & * \\
\hline Teste F Adubação & * & ns & ** & ns & ** & ns & ns & ns & ns \\
\hline Teste F (Ano X Adubação) & ns & ns & ns & ns & ns & ns & ns & ns & ns \\
\hline C. V. $(\%)$ & 22,7 & 27,2 & 20,5 & 24,6 & 25,7 & 24,3 & 30,3 & 27,3 & 34,9 \\
\hline
\end{tabular}

* **, ns Significativo a $5 \%$ e $1 \%$ de probabilidade e não significativo, respectivamente

Médias seguidas da mesma letra na mesma coluna, não diferem significativamente pelo teste de Tukey, a $5 \%$ de probabilidade 
positiva e significativamente pela acumulação de nutrientes nos grãos, com exceção do Fe. Os maiores valores dos coeficientes de determinação foram do $\mathrm{P}\left(\mathrm{R}^{2}=0,9273^{* *}\right)$ e do $\mathrm{N}\left(\mathrm{R}^{2}=0,8630^{* *}\right)$, indicando que esses nutrientes são mais importantes no aumento da produtividade em arroz irrigado. Fageria et al. (2003) mostraram que N e P são os nutri- entes que mais limitam a produtividade de arroz irrigado em solo de várzea do Brasil.

Os resultados das análises do solo após a colheita do experimento 1, foram correlacionados com a produtividade de grãos (Tabela 7), enquanto o P e o Mn foram, relacionados positiva e significativamente, com a produtividade de grãos,

Tabela 5. Acumulação de nutrientes nos grãos do arroz irrigado em diferentes tratamentos de adubação. Os valores são média de dois cultivos. Experimento 1

\begin{tabular}{|c|c|c|c|c|c|c|c|c|c|}
\hline \multirow{2}{*}{ Tratamentos } & $\mathrm{N}$ & $\mathbf{P}$ & $\mathrm{K}$ & $\mathrm{Ca}$ & $\mathrm{Mg}$ & $\mathrm{Zn}$ & $\mathrm{Cu}$ & Mn & $\mathrm{Fe}$ \\
\hline & \multicolumn{5}{|c|}{$\mathrm{kg} \mathrm{ha}^{-1}$} & \multicolumn{4}{|c|}{$\mathrm{g} \mathrm{ha}^{-1}$} \\
\hline Ad.V. $+\mathrm{N}_{0} \mathrm{P}_{0} \mathrm{~K}_{0}$ & $44,2 \mathrm{~b}$ & 11,1 & 12,7 & 4,4 & 5,6 & 172,3 & 121,8 & 1469,2 & 653,2 \\
\hline Ad.V. $+\mathrm{N}_{100} \mathrm{P}_{120} \mathrm{~K}_{100}$ & $50,5 \mathrm{ab}$ & 11,7 & 9,8 & 4,1 & 5,6 & 160,7 & 109,0 & 1685,3 & 850,8 \\
\hline Ad.V. $+N_{50} P_{60} K_{50}$ & $50,2 a b$ & 11,6 & 10,1 & 3,9 & 5,8 & 165,7 & 110,3 & 1793,5 & 661,0 \\
\hline Ad.V. $+\mathrm{N}_{75} \mathrm{P}_{90} \mathrm{~K}_{75}$ & $55,7 a b c$ & 12,4 & 11,3 & 4,4 & 6,2 & 182,7 & 121,8 & 1906,7 & 748,7 \\
\hline Ad.V. $+\mathrm{N}_{45} \mathrm{P}_{120} \mathrm{~K}_{100}$ (N, P e K no plantio) & $52,2 a b c$ & 11,6 & 10,3 & 3,9 & 5,8 & 165,5 & 110,7 & 1704,5 & 774,7 \\
\hline Ad. V. $+\mathrm{N}_{45} \mathrm{P}_{120} \mathrm{~K}_{100}$ (P e K no plantio e $\mathrm{N}$ em cobertura) & $59,2 \mathrm{c}$ & 13,3 & 11,8 & 4,6 & 6,4 & 184,0 & 118,7 & 2019,7 & 785,5 \\
\hline Ad.V. $+\mathrm{N}_{100} \mathrm{P}_{120} \mathrm{~K}_{100}+4 \mathrm{t}$ calcário ha ${ }^{-1}$ & $50,2 \mathrm{ab}$ & 11,2 & 9,7 & 3,7 & 5,4 & 161,2 & 110,5 & 1617,0 & 838,5 \\
\hline Sem Ad. V. $+\mathrm{N}_{100} \mathrm{P}_{120} \mathrm{~K}_{100}$ & $47,8 \mathrm{ab}$ & 11,2 & 9,7 & 4,2 & 5,5 & 162,0 & 113,8 & 1574,0 & 682,0 \\
\hline Média & 51,3 & 11,8 & 10,7 & 4,2 & 5,8 & 169,3 & 114,6 & 1721,2 & 749,3 \\
\hline Teste F Ano & $\star *$ & ns & ns & $\star \star$ & $\star \star$ & $\star \star$ & $\mathrm{ns}$ & ** & $\star \star$ \\
\hline Teste F Adubação & * & ns & ns & ns & ns & ns & ns & ns & ns \\
\hline Teste F (Ano X Adubação) & ns & ns & ns & ns & ns & ns & ns & ns & ns \\
\hline C. V. $(\%)$ & 12,7 & 13,9 & 12,1 & 22,7 & 13,7 & 17,1 & 22,7 & 17,1 & 26,7 \\
\hline
\end{tabular}

*, **, ns Significativo a $5 \%$ e $1 \%$ de probabilidade e não significativo, respectivamente

Médias seguidas pela mesma letra na mesma coluna, não diferem significativamente pelo teste de Tukey, a $5 \%$ de probabilidade

Tabela 6. Relação entre produtividade de grãos $(Y)$ e acumulação de nutrientes nos grãos do arroz irrigado. Os valores são médias de dois cultivos no Experimento 1

\begin{tabular}{|c|c|c|c|}
\hline Nutriente & Regressão & $\mathrm{R}^{2}$ & Valor para produtividade máxima de grãos \\
\hline $\mathrm{N}\left(\mathrm{kg} \mathrm{ha}^{-1}\right)$ & $Y=847,21+84,22 X$ & 0,8630 ** & 59,2 \\
\hline$P\left(k h^{-1}\right)$ & $Y=459,14+394,89 X$ & $0,9273^{* *}$ & 13,5 \\
\hline $\mathrm{K}\left(\mathrm{kg} \mathrm{ha}^{-1}\right)$ & $Y=-9006,96+2357,51 x-94,92 X^{2}$ & $0,5841 * *$ & 12,4 \\
\hline $\mathrm{Ca}\left(\mathrm{kg} \mathrm{ha}^{-1}\right)$ & $Y=110438,66+6960,87 X-752,20 X^{2}$ & $0,7473^{\star *}$ & 4,6 \\
\hline $\operatorname{Mg}\left(\mathrm{kg} \mathrm{ha}^{-1}\right)$ & $Y=506,24+798,35 X$ & $0,8401 * *$ & 6,5 \\
\hline Zn $\left(\mathrm{g} \mathrm{ha}^{-1}\right)$ & $Y=-4149,89+88,02 X-0,1922 X^{2}$ & $0,6691 * *$ & 228,9 \\
\hline $\mathrm{Cu}\left(\mathrm{g} \mathrm{ha}^{-1}\right)$ & $Y=-4470,08+147,18 X-0,5403 X^{2}$ & $0,5464^{* *}$ & 136,2 \\
\hline $\operatorname{Mn}\left(\mathrm{g} \mathrm{ha}^{-1}\right)$ & $Y=2093,41+1,63 X$ & $0,7413^{\star *}$ & 2253,0 \\
\hline $\mathrm{Fe}\left(\mathrm{g} \mathrm{ha}^{-1}\right)$ & $Y=1164,47+9,54 X-0,0054 X^{2}$ & $0,2057 \mathrm{~ns}$ & 883,3 \\
\hline
\end{tabular}

**, ns Significativo a $1 \%$ de probabilidade e não significativo, respectivamente. 0 valor para produtividade máxima foi calculado com base na regressão quadrática e onde a regressão foi linear, o valor médio máximo entre os oito tratamentos foi considerado adequado. Da mesma maneira, onde a regressão não foi significativa o valor médio dos oito tratamentos foi considerado adequado

Tabela 7. Relação entre produtividade de grãos (Y) e propriedades químicas do solo após a colheita do arroz irrigado. Os valores são médias de dois cultivos no Experimento 1

\begin{tabular}{llrr}
\hline Propriedades do solo & Regressão & \multicolumn{1}{c}{$\mathbf{R}^{2}$} & Valor para produtividade máxima de grãos \\
$\mathrm{pH}$ & $\mathrm{Y}=-27803,16+10312,12 \mathrm{X}-801,38 \mathrm{X}^{2}$ & $0,0381 \mathrm{~ns}$ & 5,9 \\
$\mathrm{Ca}\left(\mathrm{mmol}_{\mathrm{c}} \mathrm{dm}^{-3}\right)$ & $\mathrm{Y}=6684,39-254,73 \mathrm{X}+9,38 \mathrm{X}^{2}$ & $0,0356 \mathrm{~ns}$ & 17,2 \\
$\mathrm{Mg}\left(\mathrm{mmol}_{\mathrm{c}} \mathrm{dm}^{-3}\right)$ & $\mathrm{Y}=3428,33+393,21 \mathrm{X}-21,84 \mathrm{X}^{2}$ & $0,0093 \mathrm{~ns}$ & 9,4 \\
$\mathrm{Al}\left(\mathrm{mmol}_{\mathrm{c}} \mathrm{dm}^{-3}\right)$ & $\mathrm{Y}=5390,31-118,88 \mathrm{X}$ & $0,0457 \mathrm{~ns}$ & 1,9 \\
$\mathrm{H}+\mathrm{Al}\left(\mathrm{mmol}_{\mathrm{c}} \mathrm{dm}^{-3}\right)$ & $\mathrm{Y}=7525,99-59,89 \mathrm{X}$ & $0,1045 \mathrm{~ns}$ & 39,5 \\
$\mathrm{P}\left(\mathrm{mg} \mathrm{dm}^{-3}\right)$ & $\mathrm{Y}=3888,72+90,32 \mathrm{X}$ & $0,2581^{*}$ & 17,7 \\
$\mathrm{~K}\left(\mathrm{mg} \mathrm{dm}^{-3}\right)$ & $\mathrm{Y}=4184,37+12,85 \mathrm{X}$ & $0,0830 \mathrm{~ns}$ & 92,7 \\
$\mathrm{Zn}\left(\mathrm{mg} \mathrm{dm}^{-3}\right)$ & $\mathrm{Y}=2899,39+1207,82 \mathrm{X}-109,96 \mathrm{X}^{2}$ & $0,1407 \mathrm{~ns}$ & 2,4 \\
$\mathrm{Cu}\left(\mathrm{mg} \mathrm{dm}^{-3}\right)$ & $\mathrm{Y}=21772,43-16861,19 \mathrm{X}+4168,57 \mathrm{X}^{2}$ & $0,4755^{\star *}$ & 2,3 \\
$\mathrm{Fe}\left(\mathrm{mg} \mathrm{dm}^{-3}\right)$ & $\mathrm{Y}=-4152,01+22,88 \mathrm{X}-0,0138 \mathrm{X}^{2}$ & $0,1352 \mathrm{~ns}$ & 763,4 \\
$\mathrm{Mn}\left(\mathrm{mg} \mathrm{dm}^{-3}\right)$ & $\mathrm{Y}=-12854,46+709,83 \mathrm{X}-6,86 \mathrm{X}^{2}$ & $0,3756^{* *}$ & 51,7 \\
$\mathrm{M} .0\left(\mathrm{~g} \mathrm{dm}^{-1}\right)$ & $\mathrm{Y}=-17535,21+2985,53 \mathrm{X}-97,75 \mathrm{X}^{2}$ & $0,0297 \mathrm{~ns}$ & 14,7 \\
CTC $\left(\mathrm{mmol}_{\mathrm{c}} \mathrm{dm}^{-3}\right)$ & $\mathrm{Y}=-15667,81+641,54 \mathrm{X}-4,92 \mathrm{X}^{2}$ & $0,0361 \mathrm{~ns}$ & 67,6 \\
Sat. por bases $(\%)$ & $\mathrm{Y}=3264,51+61,23 \mathrm{X}-0,3724 \mathrm{X}^{2}$ & $0,0522 \mathrm{~ns}$ & 41,6 \\
\hline
\end{tabular}

*, **, ns Significativo a $5 \%$ e $1 \%$ de probabilidade e não significativo, respectivamente. Os valores para produtividade máxima foram calculados com base em equações de regressão, em que $\mathrm{R}^{2}$ foi significativo e, nos casos em que $\mathrm{R}^{2}$ não foi significativo, 0 valor médio dos tratamentos foi considerado adequado. Em caso de regressão positiva linear, 0 valor médio máximo e, em caso de regressão linear negativa, o valor médio mínimo foi considerado adequado para produtividade máxima 
significando que esses dois nutrientes estavam mais deficientes no solo, na época da colheita. Na literatura não existem muitos dados de pesquisa para se fazer comparação com os resultados obtidos neste trabalho, os valores de outras propriedades químicas apresentados para produtividade máxima de grãos podem servir como referência na interpretação de análise de solo várzea para arroz irrigado.

\section{CONCLUSÕES}

1. O uso de adubo verde e químico aumenta a produtividade de grãos.

2. A produtividade máxima de grãos é obtida com o tratamento de adubo verde $+45 \mathrm{~kg} \mathrm{~N} \mathrm{ha}^{-1}, 120 \mathrm{~kg} \mathrm{P}_{2} \mathrm{O}_{5}$ ha $^{-1} \mathrm{e}$ $100 \mathrm{~kg} \mathrm{~K}_{2} \mathrm{O}$ ha $^{-1}$ (P e K no plantio e $\mathrm{N}$ em cobertura no estádio de perfilhamento ativo).

3. Entre os componentes da produtividade, o comprimento da panícula é influenciado positiva e significativamente pela adubação.

4. A acumulação de nutrientes nos grãos apresenta correlação positiva e significativa com a produtividade, com exceção do Fe.

5. Os teores de $\mathrm{P}, \mathrm{Cu}$ e $\mathrm{Mn}$ no solo são mais limitantes da produtividade de grãos de arroz irrigado em solo de várzea.

\section{LITERATURA CITADA}

Balasubramaniyan, P.; Palaniappan, S. P. Green manure (GM) management and its effect on lowland rice yield. International Rice Research Newsletter, Los Baños, v.17, p.20-21, 1992.

EMBRAPA - Empresa Brasileira de Pesquisa Agropecuária. Serviço Nacional de Levantamento e Conservação de Solos (Rio de Janeiro). Manual de métodos de análise de solo. 2.ed. Rio de Janeiro: Embrapa CNPS, 1997, 212p.

Fageria, N. K. Resposta do arroz irrigado à adubação residual e aos níveis de adubação em solo de várzea. Revista Brasileira de Engenharia Agrícola e Ambiental, Campina Grande, v.4, n.2, p.177-182, 2000.
Fageria, N. K.; Baligar, V. C. Response of lowland rice and common bean grown in rotation to soil fertility levels on a várzea soil. Fertilizer Research, Dordrecht, v.45, p.13-20, 1996.

Fageria, N. K.; Slaton, N. A.; Baligar, V. C. Nutrient management for improving lowland rice productivity and sustainability. Advances in Agronomy, New York, v.80, p.63-152, 2003.

Fageria, N. K.; Stone, L. F.; Santos, A. B. dos. Maximização da eficiência de produção das culturas. Brasília: Embrapa Comunicação para Transferência de Tecnologia; Santo Antônio de Goiás: Embrapa Arroz e Feijão, 1999. 294p.

George, T.; Ladha, J. K.; Garrity, D. P.; Buresh, R. J. Legumes as nitrate catch crops during the dry-to-wet transition in lowland rice cropping systems. Agronomy Journal, Madison, v.86, p.267-273, 1994.

Gravois, K. A.; Helms, R. S. Path analysis of rice yield and yield components as affected by seeding rate. Agronomy Journal., Madison, v.84, n.1, p.1-4, 1992.

Hasegawa, T.; Koroda, Y.; Seligman, G.; Horie, T. Response of spikelet number to plant nitrogen concentration and dry weight in paddy rice. Agronomy. Journal, Madison, v.86, n.4, p.673-676, 1994.

Kolar, J. S.; Grewal, H. S.; Singh, B. Nitrogen substitution and higher productivity of a rice-wheat cropping system through green manuring. Tropical Agriculture, Trinidad, v.70, p.301-304, 1993.

Mariot, C. H. P.; Silva, P. R. F. da; Menezes, V. G.; Teichmann, L. L. Resposta de duas cultivares de arroz irrigado à densidade de semeadura e à adubação nitrogenada. Pesquisa Agropecuária Brasileira, Brasília, v.38, p.233-241, 2003.

Moraes, J. F. V; Rabelo, N. A. Um método simples de digestão de plantas. Goiânia: Embrapa CNPAF, 1986. Documentos, 12

Raij, B. van. Fertilidade do solo e adubação. Piracicaba: Potafos, 1991. 343p.

Santos, A. B. dos; Fageria, N. K.; Zimmermann, F. J. P. Atributos químicos do solo afetado pelo manejo da água e do fertilizante potássico na cultura de arroz irrigado. Revista Brasileira de Engenharia Agrícola e Ambiental, Campina Grande, v.6, p.12-16, 2002.

Singh. Y.; Khind, C. S.; Singh, B. Efficient management of leguminous green manure's in wetland rice. Advances in Agronomy, New York, v.45, p.135-189, 1991. 